\title{
TMS-EEG co-registration: On TMS-induced artifact
}

\author{
Domenica Veniero, Marta Bortoletto, Carlo Miniussi * \\ Department of Biomedical Sciences and Biotechnology, National Institute of Neuroscience, University of Brescia, Viale Europa 11, 25123 Brescia, Italy \\ Cognitive Neuroscience Section, IRCCS San Giovanni di Dio Fatebenefratelli, Via Pilastroni 4, 25125 Brescia, Italy
}

See Editorial, pages 1225-1226

\section{A R T I C L E I N F O}

\section{Article history:}

Accepted 2 April 2009

Available online 16 June 2009

\section{Keywords:}

Transcranial Magnetic Stimulation

Electroencephalography

Artifact

Electrode

TMS-evoked potential

TEP

\begin{abstract}
A B S T R A C T
Objective: The combination of brain stimulation by transcranial magnetic stimulation (TMS) and simultaneous electroencephalographic (EEG) recording has the potential to be of great value for understanding human brain functions. Recording EEG during TMS can be technically challenging because TMS induces a very strong electrical field that can saturate recording amplifiers for a long duration. Advances in amplifier technology, however, have led to the development of TMS-compatible EEG equipment that can work in very high, time-varying magnetic fields without saturation. The aim of the present study was to identify stimulus-related artifacts, and to provide experimental data containing the length of the artifact induced by the magnetic field and its variations with respect to the experimental setting.

Methods: A phantom head was stimulated to record the artifact while excluding cortical responses. We tested different types of electrodes, coils, models of stimulator, and frequencies and intensities of stimulation to see how these parameters influence the duration of the artifact.

Results: The electrical artifact produced by the magnetic pulse lasted approximately 5 ms following TMS onset. Its length was invariant irrespective of different experimental conditions.

Conclusions: These data suggest that it is possible to analyze the cortical evoked response induced by TMS 5 ms after TMS onset.

Significance: The possibility to study the early physiological responses to TMS stimulation may have valuable implications for both clinical and experimental purposes, providing information about the early direct cortical response of the stimulated areas.

(C) 2009 International Federation of Clinical Neurophysiology. Published by Elsevier Ireland Ltd. All rights reserved.
\end{abstract}

\section{Introduction}

In recent years, a new generation of amplifiers has been introduced that permits the co-registration of electroencephalographic (EEG) activity, which has a temporal resolution of a few milliseconds, during Transcranial Magnetic Stimulation (TMS). This TMSEEG co-registration provides valuable information about the characteristics of cortical reactivity and connectivity in response to magnetic stimulation (Ilmoniemi et al., 1997; Komssi and Kahkonen, 2006), and how functional activity links to behavior through the study of TMS-induced modulations (Miniussi and Thut, 2009). Moreover, EEG can be used to study how TMS interacts with rhythmic brain activity, and vice versa, as well as how

\footnotetext{
* Corresponding author. Address: Department of Biomedical Sciences and Biotechnology, National Institute of Neuroscience, University of Brescia, Viale Europa 11, 25123 Brescia, Italy. Tel.: +39 0303717441 ; fax: +39 0303717443.

E-mail address: miniussi@med.unibs.it (C. Miniussi).
}

rhythmic brain stimulation can be used to modify brain functions (Thut and Miniussi, 2009).

In TMS-EEG co-registration procedure, a sample-and-hold circuit that is controlled by the initiation of the TMS pulse and locks the EEG signal is typically used. The signal is held for a few milliseconds immediately following TMS (Iramina and Maeno, 2003; Taylor et al., 2007; Virtanen et al., 1999), thereby avoiding saturation of the recording amplifiers by the magnetic stimuli and allowing the recording of EEG activity to take place quite early in response to TMS.

Nevertheless, this methodology poses some problems. The first is that the TMS pulse only lasts for an extremely short time - less than $1 \mathrm{~ms}$ (approximately $300 \mu \mathrm{s}$ ), and blocking of the amplifiers does not allow for the opportunity to see what happens during or immediately after the TMS pulse. The second problem is that this method compares the level of the signal before the TMS pulse (i.e., normalized baseline activity at each site is supposed to be at $0 \mu \mathrm{V}$ ) to the level soon after TMS that we expect to be affected by currents induced at the cortical level. Studies on EEG could 
emphasize the comparison of transient activity engaged by TMSevoked responses (TEPs) to a control condition. The data are interpreted in terms of relative differences. Nevertheless, this option eliminates the possibility to study the initial contributing effects in the level or distribution of brain activity immediately after TMS onset. It is possible, and indeed likely, that real changes in levels of activation happen in this interval, and these changes are likely to be correlated with the peripheral response (e.g., motor evoked potential - MEP).

New TMS-compatible EEG equipment can work in very high time-varying magnetic fields without saturating the amplifier. It is therefore possible to run continuous EEG recordings during TMS stimulation. Recently, Bonato et al. (2006) used a system that does not make use of particular devices to pin the amplifier output to a constant level during and after stimulation. Using this method, they were able to observe the temporal variation and spatial distribution of the TEP even during the TMS pulse.

Even this new technology poses some problems. Because the TMS pulse has a very high-energy component, it most likely induces eddy currents that last longer than the TMS pulse. At least part of the initial large response recorded after TMS stimulation is therefore most likely due to non-cortical currents induced by the magnetic field. It appears increasingly important to characterize the TMS-induced artifactual activity in order to disentangle it from the EEG activity.

In several recent papers, it was reported that it is not possible to record a clear signal before 30-300 ms after stimulation (Bender et al., 2005; Morbidi et al., 2007; Taylor et al., 2008; Thut et al., 2003). Subtraction approaches have been proposed to resolve this issue (Thut et al., 2003). To control for the processing reflecting TMS-induced artifacts, data from a control condition using a phantom (Bender et al., 2005) or with TMS and no task (Thut et al., 2003) were used to build a template that was subtracted from data with TMS and a task. The cases in which electrodes are irremediably influenced by the artifact were excluded from further analyses (Komssi et al., 2004). As an alternative solution, the use of filtering (Morbidi et al., 2007) or artifact correction (Litvak et al., 2007) to remove TMS-induced artifacts has also been proposed. Artifact correction implies an a priori assumption of artifactual activity based on models. Even if independent component analysis is an optimal solution to perform a blind separation of EEG sources, it may not be the ideal method to dissociate cortical activity from artifactual TMS-related activity.

Several reasons can account for the long-lasting TMS artifact. The main one may be that electrodes and skin have magnetic properties, and they may therefore be affected by the TMS pulse and generate an extra-cortical signal in the recording.

Recording of EEG is traditionally performed with electrodes made of tin, silver, silver-chloride or gold, with a fairly large and ring shaped surface that permits eddy currents to be generated during TMS. The TMS pulse can cause heating or even movement of the electrodes. In terms of safety, this poses a risk of burning of the tissue under the electrode (Pascual-Leone et al., 1990, 1993; Roth et al., 1992; Wassermann, 1998). In terms of recording, heating and movement can induce electrical artifacts that prevent the recording of adequate signal. To minimize overheating of the electrodes located in the vicinity of the stimulating coil, it is possible to cut a section out of the ring metal electrodes (Roth et al., 1992), thereby creating a radial notch. Plastic electrodes have also been used (Ives et al., 2006), but with a conductive-silver epoxy coat. Moreover, it has been shown that by using TMS-compatible electrodes, it is possible to obtain the same result without cutting the electrodes (Bonato et al., 2006; Virtanen et al., 1999).

Thus far, it is not clear what the best technical conditions to record such a signal are. Whether the ideal electrodes must to be cut in a ring shape, whether there is only an initial artifact soon after the TMS pulse, if longer latency artifacts are present (Moliadze et al., 2003) and, most importantly of all, how long the TMS-induced artifact lasts, remains to be elucidated. In short, it remains unclear how the intrinsic properties of electrodes, amplifiers and parameters of stimulation contribute to artifacts in the recorded signal.

In the present study, we provide experimental data describing the characteristics of the artifact duration induced by the magnetic field using a commercial system. We employed a phantom 'head' that was stimulated so the artifact could be recorded while excluding any cortical responses. We tested different types of electrodes, coils, model of stimulators, frequency and intensity of stimulation to evaluate how these parameters influenced the duration of the artifact. Moreover, since TMS artifacts in the EEG depend on the capacitive properties of the skin (Julkunen et al., 2008), we also stimulated a part of the body (the knee) so we could evaluate the artifact in a model with similar properties of skin but still avoiding any cortical response. Finally, all control conditions were compared to a cortical stimulation.

\section{Materials and methods}

TMS-EEG co-registration was performed on three models: a phantom head made with a Cucumis melo (i.e., a cantaloupe melon, of about the dimension of a small head), a knee and the head of a healthy volunteer. The melon was used to test whether TMS artifact varies with different TMS and EEG recording parameters. To this aim any material with stable electrical properties is suitable. Fruit and vegetable skin has been shown to share dielectric properties with human skin (Freeston and Tozer, 1995; Holder et al., 1996; Tidswell et al., 2003). The dielectric constant $\left(\varepsilon^{\prime}\right)$ of melons measured from the surface $\left(\varepsilon^{\prime}=30-50\right.$; frequency range $200 \mathrm{MHz}-2 \mathrm{GHz}, 24^{\circ} \mathrm{C}$ ) (Nelson et al., 2008) and the dielectric constant of human skin $\left(\varepsilon^{\prime}=40-50\right.$; frequency range 200$500 \mathrm{MHz}, 36^{\circ} \mathrm{C}$ ) (Sunaga et al., 2002) are comparable. Moreover melon pulp has higher dielectric constant than its skin (Nelson et al., 2008). The same differences can be identified as regards grey matter and muscle in comparison to human skin (Young et al., 2002). However, electric properties of materials are complex and vary depending on frequency of the applied field, temperature, and other parameters. Consequently, we controlled for the electric properties of the human skin by recording the induced artifact from a knee. As a final control we also stimulated the head of a healthy volunteer so that we could compare the responses obtained for each model.

\subsection{Stimulation}

The TMS was delivered over electrodes on a phantom head using the Magstim Super Rapid ( $50 \mathrm{~Hz}$ - biphasic, four boosters), Magstim Standard Rapid ${ }^{2}$ ( $50 \mathrm{~Hz}$ - biphasic - single Power Supply Module) and a Bistim (monophasic) systems. (We will refer to stimulators as Super Rapid, Standard Rapid ${ }^{2}$ and Bistim, respectively.) Four figure-of-eight coils were used: standard double $70 \mathrm{~mm}$, custom double $70 \mathrm{~mm}$, custom double $50 \mathrm{~mm}$ and custom double $25 \mathrm{~mm}$ (Magstim Company Limited, Whitland, UK). Two stimulation conditions were used for the sham-TMS. In the first, the Magstim Placebo Coil double $70 \mathrm{~mm}$ was employed. This coil was designed to replicate the standard figure-of-eight coil, and provides discharge without stimulating cortical tissues since its magnetic field output is approximately 10 -fold lower than that delivered by the standard coil (maximal magnetic field strength $0.2 \mathrm{~T}$ ). The other sham condition was performed with a real coil that was turned over and a $30 \mathrm{~mm}$-thick plywood shield of the same shape and size was fastened to the coil and placed against the electrode (Rossi et al., 2007). 
To reduce the variability of the induced artifacts over time, the stimulating coil was fixed by means of a mechanical support that consisted of an articulated mechanical holding arm (Magic arm Manfrotto with two super clamps) and a heavy duty tripod for all conditions. This aid arm allowed maximum flexibility for positioning the coil at the desired location, orientation and maximum stability once fixated. The lower surface of the coil was held approximately $1 \mathrm{~mm}$ from the stimulating electrode and it was continuously monitored by the investigator.

Magnetic stimuli were delivered at different intensities and frequencies. The intensity ranged from $10 \%$ to $100 \%$ of the stimulator output to verify how these parameters influence the artifact. Moreover, a single pulse and two stimulation frequencies ( 5 and $20 \mathrm{~Hz}$ ) were applied. The exact values used are listed below in Sections 2.3 and 3.

\subsection{EEG recordings and analysis}

For recording of the signal, TMS-compatible EEG equipment (BrainAmp 32 MR plus or BrainAmp DC, BrainProducts $\mathrm{GmbH}, \mathrm{Mu}-$ nich, Germany) was used. The design of the BrainAmp amplifier allows the fine adaptation to the TMS stimulus magnitude by the ability to adjust the amplifier sensitivity and operational range. This was done by using a sensitivity of $100 \mathrm{nV} /$ bit (Signal range/resolution) and an Analog/Digital conversion range of $6553.5 \mu \mathrm{V}( \pm 3.277 \mathrm{mV})$, which was generally sufficient to prevent saturation under the given stimulus conditions (as an alternative, it is possible to use a sensitivity of $500 \mathrm{nV} /$ bit $\pm 16.385 \mathrm{mV}$ ). A continuous recording mode without the use of any sample and hold circuits was chosen. The signal was band-pass filtered at $0.01-1000 \mathrm{~Hz}$ and digitized at a sampling rate of $5000 \mathrm{~Hz}$. A low impedance mode was employed.

In all sessions, unless explicitly stated, the signal was recorded from a set of TMS-compatible sintered $\mathrm{Ag} / \mathrm{AgCl}$ electrodes (Easy cap $\mathrm{GmbH}$, Munich, Germany) consisting of rings of $2 \mathrm{~mm}$ thickness, with inner and outer diameters of $6 \mathrm{~mm}$ and $12 \mathrm{~mm}$, respectively. Additional recordings were done only under the $20 \mathrm{~Hz}$ condition (see below) with small sintered $\mathrm{Ag} / \mathrm{AgCl}$ disks that were $1 \mathrm{~mm}$ thick and $3 \mathrm{~mm}$ in diameter, mounted in an elastic cap.

Three electrodes were placed at the center of our phantom and two were placed as temporal recording sites, that is, on the curvature of the phantom. Two additional electrodes were used as ground (rostral) and reference (caudal) with respect to the coil handle. Contact impedance was maintained below $5 \mathrm{k} \Omega$ (range $0-3 \mathrm{k} \Omega$ ). In one experimental condition, we modified the used electrodes to verify variations in electrode response characteristics (details below). Temperature variations were also measured by means of infrared thermometer. The epoching of TMS-related EEG was performed off-line, from $10 \mathrm{~ms}$ before to $100 \mathrm{~ms}$ after TMS onset. Firstly, in order to define the artifact duration the entire EEG epoch (from -10 to $100 \mathrm{~ms}$ ) was considered for the analysis. Subsequently, the time window was reduced around the magnetic pulse since no other response was identified in the whole epoch. This criterion was kept constant for the phantom and the knee. Cortical stimulation was therefore characterized around the same window. In such a way we also tested the possibility to record cortical responses in a time window usually left out for technical reasons.

Moreover, a Fast Fourier Transform (FFT) was performed on 10 epochs of $2 \mathrm{~s}$ preceding and 10 epochs of $2 \mathrm{~s}$ (starting at $5 \mathrm{~ms}$ after TMS onset) following magnetic stimulus. For the FFT analyses, the sampling rate was changed to reduce the number of data points to 8192. Transformation was performed for each electrode using a Hamming window with the resolution of $0.5 \mathrm{~Hz}$. Finally, FFT epochs were averaged for each condition $(20 \mathrm{~Hz}$ session: $30 \%$, $40 \%, 50 \%$ of maximum stimulator output - MSO; single pulse session: $30 \%$ of MSO see below).
Recordings were processed using the Brain Vision Analyzer (Brain Products GmbH, Munich, Germany).

\subsection{Procedure}

\subsubsection{Single pulse TMS}

We first tested the differences between biphasic and monophasic stimulators (Super Rapid and Bistim, respectively) with a standard double $70-\mathrm{mm}$ coil. The center of the junction of the coil wings was centered on one electrode. Subsequently, using the biphasic stimulator, different stimulation intensities of $30 \%, 40 \%$, 50\%, 60\% MSO were evaluated. Finally, all coils mentioned above, including the two different sham coils, were tested with the biphasic stimulator.

\subsection{2. $5 \mathrm{~Hz} T M S$}

Sixty stimuli per condition were delivered with the biphasic stimulators (Super Rapid and Standard Rapid ${ }^{2}$ ) in six trains of 10 stimuli each and a $5 \mathrm{~Hz}$ repetition rate with an inter-train-interval of $14.5 \mathrm{~s}$. A standard double $70-\mathrm{mm}$ coil was used. The influence of stimulation intensity was systematically tested from $10 \%$ to $100 \%$ of MSO increasing in steps of $10 \%$. Additional recordings at $40 \%$ of MSO, with the Super Rapid stimulator were made varying the number of boosters during the stimulation (from 1 to 4 ) with and without a voltage stabilizer to see if variation of "late" artifact could be obtained (see Section 3 and figures).

\subsection{3. $20 \mathrm{~Hz} T M S$}

In each condition, 100 stimuli were delivered in 10 trains of 10 stimuli each with a $20 \mathrm{~Hz}$ repetition rate and an inter-train-interval of $14.5 \mathrm{~s}$. A standard double $70-\mathrm{mm}$ coil was used with the biphasic stimulators. The interaction between high frequency and stimulation intensity was tested at $30 \%, 40 \%$, and $55 \%$ of MSO to verify the presence of summation of artifact effects between stimuli.

Additionally, to address the problem of how electrode shape or type (disk vs. ring) affects the artifact length and amplitude, four types of electrodes were tested at $30 \%, 40 \%$, and $55 \%$ of MSO. Two of the ring electrodes were "o-shape" sintered electrodes, two were sintered electrodes with a $2 \mathrm{~mm}$ slit in the ring "c-shape" and two electrodes had the slit closed by means of silicone "closed c-shape". To assess the potential effect of different kinds of electrodes, our stimulation site was chosen to deliver the stimulus at three recording sites, each one of a different type (o-shape, c-shape, closed c-shape). In other words, three electrodes were arranged to be equidistant below the center of the coil.

\subsection{Knee stimulation}

In this session, we performed stimulation on the patella to test the artifact in a model including skin properties. We tested the effect of impedance on the artifact features by performing a stimulation with high impedance values (range: $21-25 \mathrm{k} \Omega$ ) and with low impedance values (range: $0-3 \mathrm{k} \Omega$ ). Forty trains of 10 stimuli were delivered for a total of 400 stimuli with the biphasic stimulator at a frequency of both 5 and $20 \mathrm{~Hz}$ using a standard double 70 $\mathrm{mm}$ coil at $50 \%$ of MSO.

\subsection{Cortical stimulation}

To compare the results obtained from the phantom head and patella with real cortical stimulation, a TMS session was performed for one subject. The study was approved by the Local Ethical Committee and informed consent from the participant was obtained prior to the beginning of the experiment. The TMS was carried out by a biphasic stimulator connected to a $70 \mathrm{~mm}$ standard figure-of-eight coil. 
The motor hot spot was defined as the point where TMS induced the maximum motor evoked response (MEP) from the Abductor Digiti Minimi (ADM) of right hand. The resting motor threshold (RMT) was determined as the lowest stimulus intensity, which produced at least five MEPs of $50 \mu \mathrm{V}$ in the ADM muscle out of 10 consecutive stimuli (Rossini et al., 1994). The EEG was continuously acquired from 29 scalp sites (Fp1, Fp2, F7, F3, Fz, F4, F8, FC5, FC1, FC2, FC6, T7, C3, Cz, C4, T8, CP5, CP1, Cp2, Cp6, P7, P3, Pz, P4, $\mathrm{P} 8, \mathrm{O} 1, \mathrm{Oz}, \mathrm{O} 2, \mathrm{Iz}$ ) using sintered electrodes mounted on an elastic cap. The ground electrode was positioned in Fpz and the linked mastoid served as the active reference for all electrodes. Horizontal and vertical EOG eye movements were recorded from electrodes positioned on the outer canthi of both eyes and from electrodes located beneath the right eye, respectively. A total of 400 stimuli were delivered on motor hot spot at RMT (the nearest electrode to the stimulating coil was C3) in 40 trains of 10 stimuli each with a $20 \mathrm{~Hz}$ repetition rate and an inter-train-interval of $14.5 \mathrm{~s}$.

\section{Results}

\subsection{Frequency}

The stimulation frequency did not have an effect on artifact shape or duration. Indeed, in both 5 and $20 \mathrm{~Hz}$ conditions the EEG signal was back to baseline in the same time window (5$5.6 \mathrm{~ms}$ ). The results obtained with the two stimulation frequencies ( 5 vs. $20 \mathrm{~Hz}$ ) were therefore comparable. Repetitive TMS was not able to induce any modulation of the artifact amplitude or duration per se. Because of this, no summation of the induced artifacts was found. As shown in Fig. 1, variations in the artifact size between 0 and $2.2 \mathrm{~ms}$ were clearly visible, although these changes had no relation to the stimulus position in the train and are probably due to casual fluctuations of the stimulation and recording system.

\subsection{Intensity}

The TMS-induced artifact was always of the same length regardless of stimulation intensity. The shape of the artifact was constant for intensities from $10 \%$ to $60 \%$ of MSO, while for higher intensities (from $70 \%$ to $100 \%$ of MSO), the first $2 \mathrm{~ms}(0-2.5 \mathrm{~ms}$ ) were characterized by a slower recovery of the signal (i.e., higher intensities caused an increase in signal amplitude), which in turn induced a short amplifier saturation. Namely, all tested intensities produced a series of negative and positive deflections, the first one always reaching the maximum amplifier output within $2 \mathrm{~ms}$. For the intensities ranging from $10 \%$ to $60 \%$ of MSO, the amplifier saturation lasted about $1 \mathrm{~ms}$, while for all intensities ranging from $70 \%$ to $100 \%$ of MSO the first deflection was characterized by an inversed polarity and a longer recovery period, reaching 2 ms when $100 \%$ of MSO was applied. The subsequent deflections had decreasing amplitudes reaching about $10 \mu \mathrm{V}$ within $4-4.5 \mathrm{~ms}$. The signal was back to baseline $(0 \pm 2 \mu \mathrm{V})$ in 5-5.6 ms with no linear correlation between intensity and length or amplitude of artifact (Fig. 2).

\subsection{Electrodes}

We found that cut-ring electrodes (c-shape) did not reduce TMS pulse-induced artifacts or drifts, since recordings with the three types of electrodes were not different. After an initial large artifact, the signal was back to baseline $(0 \mu \mathrm{V})$ in the same amount of time

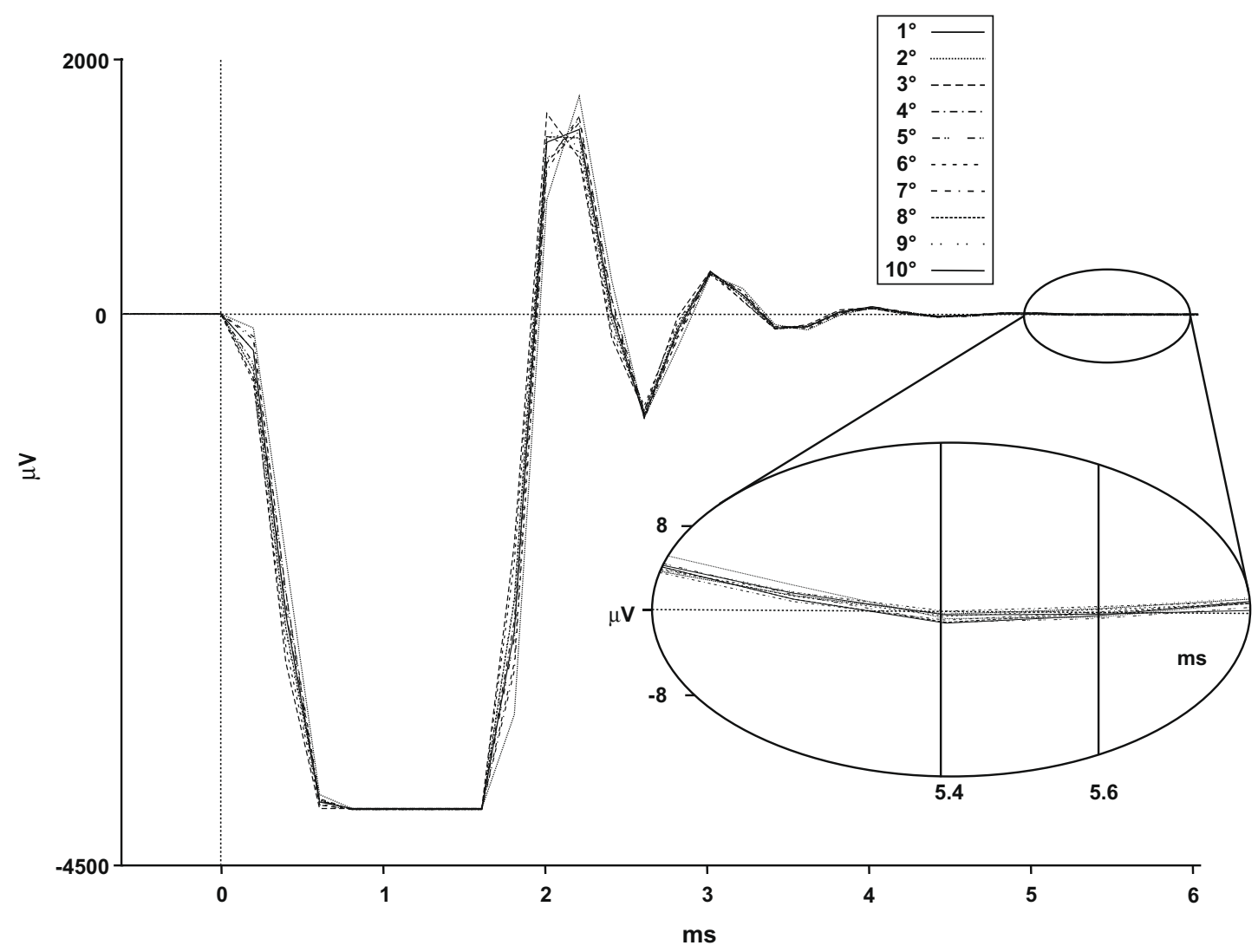

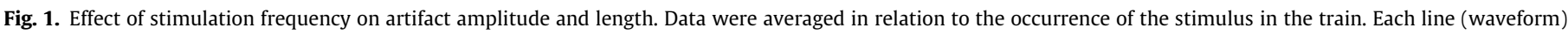

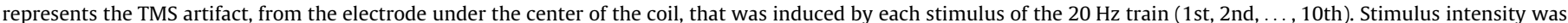

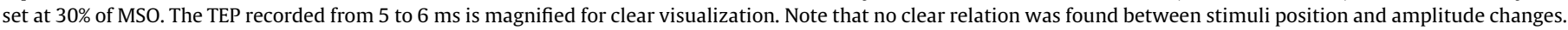
Voltage is plotted with positive values upward in this and all subsequent figures. 


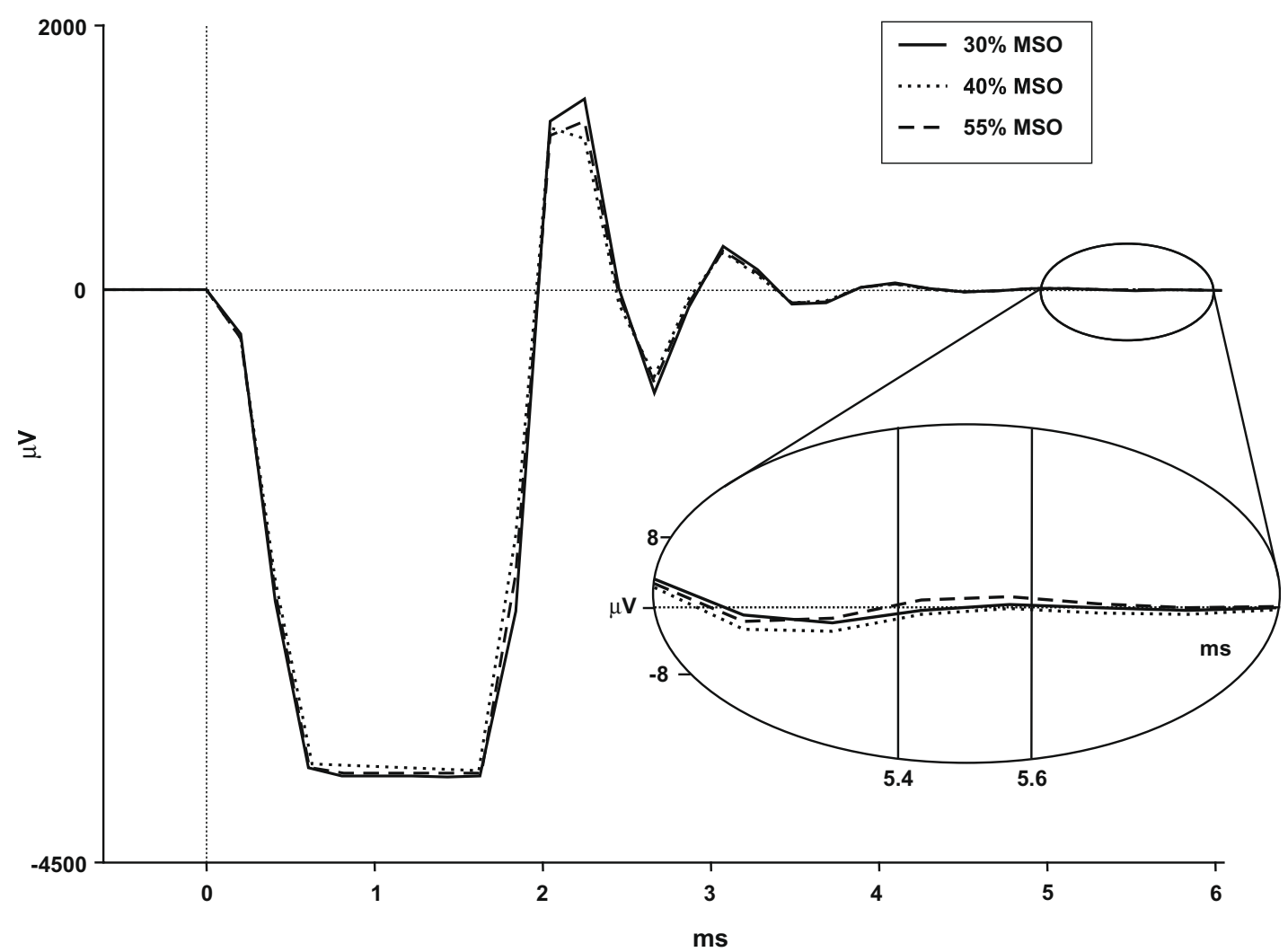

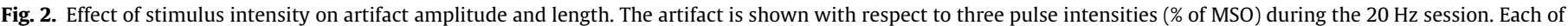
the waveforms represents the average of 100 stimuli delivered at the same intensity. The TEP recorded from 5 to 6 ms is magnified for clear visualization.

for all electrodes (approximately $5 \mathrm{~ms}$ ). Relative to the artifact shape, variations were related only to the location of the electrode with respect to the coil. In other words, there was a reduction in amplitude that roughly correlated with the distance of the recording site from the coil. The TMS evoked artifact was made up of several deflections of alternating positive and negative polarity, each having a quite stable shape and latency within a single EEG channel. When comparing different recording sites we found each channel to have a slightly different latency for each deflection. No systematic investigation was performed on this parameter because of a limited number of channels. In this session we also verified that the TMS artifact in the signal was reduced in length if the electrode wires were kept loop free and arranged in a radial way from the center of the magnetic field of the coil, thereby avoiding further electrical interferences. Finally, sintered ring electrodes were not heated by magnetic stimulation independently of their shape (cut-ring or not).

\subsection{Coil}

To understand if the TMS-induced artifact was affected by the coil features, a comparison between four types of stimulating coils was performed. Generally, results indicated that this parameter did not affect the artifact duration, despite some differences in its shape in the first millisecond after the magnetic pulse (from 0 to $2 \mathrm{~ms}$ ). The magnetic field intensity ( $\mathrm{T}$ ) depends on coil diameter, therefore at the same MSO different coils mimicked differences in output intensity (e.g., from 2.2 to $4 \mathrm{~T}$ at $100 \%$ of MSO for the 70- and 50-mm coils, respectively).

The amplitude of TMS artifact was reduced in both sham conditions, while no changes in its length were found in respect to real stimulation. Moreover, when the stimulation was performed with the placebo coil, the initial part of the induced artifact (from 0 to $2 \mathrm{~ms}$ ) was smaller compared to our self-made sham coil. This result can be explained by the strength of the magnetic field produced by the two coils. The Magstim Placebo Coil output is about 10-fold lower in respect to that delivered by the Standard Coil, while for the $30 \mathrm{~mm}$-thick plywood shield, the attenuation of the induced electric field is roughly 8-fold lower (see Rossi et al., 2007). No differences were found between the types of coils after 2 ms.

\subsection{Monophasic vs. biphasic stimulator}

The monophasic stimulator evoked a higher artifact than the biphasic stimulator. On average, the difference between the stimulators was of approximately $2000 \mu \mathrm{V}$ in the initial larger deflection and of about $200 \mu \mathrm{V}$ in the last deflection (at about $2.5 \mathrm{~ms}$ ). Nonetheless, the artifact duration was comparable between the two stimulators.

\subsection{Knee stimulation}

When patella was stimulated, we found an induced artifact comparable in length to that evoked by the stimulation of the phantom. Moreover, when trains at $20 \mathrm{~Hz}$ were delivered, no summation of the stimuli was found. These results negate the putative differences of this model that could be due to skin conduction properties.

Results indicated that at higher impedance values, differences between the artifacts recorded from different sites became greater. Specifically, in the higher impedance condition, the decay of the artifact was slower (recovering time: $15-20 \mathrm{~ms}$ ) and its amplitude was more than two times the amplitude in the lower impedance condition in the electrodes that were not directly stimulated. 


\subsection{Later artifact}

Several milliseconds after TMS pulses, the signal was contaminated by a coil recharge artifact that was present with the Super Rapid as well as with the Standard Rapid ${ }^{2}$ stimulator, but not with the Bistim. Its amplitude was constant $(\sim 12 \mu \mathrm{V})$ while the latency increased with the increase of the power strength (Fig. 3a). At 10\% of the stimulator output the latency was $8.2 \mathrm{~ms}$, and every increase of an order of $10 \%$ corresponded to an increase of approximately 3-10 ms depending on the type of stimulator (i.e., Super Rapid or Standard Rapid ${ }^{2}$ ) (Fig. 3b). Both stimulators generate a magnetic pulse with the same rise time, pulse width and peak magnetic field ( $86 \mu \mathrm{s}, 345 \mu \mathrm{s} 2.2 \mathrm{~T}$, respectively, when a standard double $70 \mathrm{~mm}$ coil is connected to the device). Nevertheless they differ in terms of power provided ( 2 vs. 4 power supply units). Since recharging time is related to power, discrepancies found were due to the different recharging times. Indeed, this artifact could be avoided only when the stimulation was performed with the Super Rapid device, which allowed the use of only one booster which caused the slowing down of the recharge and eliminated the ringing of the currents in the coil. No reduction was obtained with the voltage stabilizer.

\subsection{Fast Fourier Transform}

The FFT analysis indicated that the power spectra calculated for pre- and post-stimulus signals was not different, irrespective of stimulus intensity (30\%, 40\%, 50\% MSO) or frequency of stimulation ( $20 \mathrm{~Hz}$, single pulse). These results confirm that the EEG contamination is limited to the first few milliseconds after pulse delivery, regardless of the stimulation parameters.

\subsection{Comparison with cortical stimulation}

Cortical stimulation was performed at individual RMT (57\% of MSO). The artifact time-course was similar for cortical stimulation, patella and phantom head stimulation up to $5 \mathrm{~ms}$ (Fig. 4). Differences appeared at $5.8 \mathrm{~ms}$ after the TMS pulse when the EEG signal went back to baseline for all conditions with exception of the cortical stimulation. This showed a positive and a negative deflection, peaking at 5.8 and $8 \mathrm{~ms}$.

\section{Discussion}

We collected data using an EEG recording system that allows continuous data recording throughout TMS stimulation without saturation of the signals and does not require pinning the preamplifier output to a constant level during TMS delivery. The aim was to identify the best technical conditions to minimize stimulus-related artifacts and provide experimental data regarding the artifact length.

The electrical artifact produced by the magnetic pulse lasted approximately $5 \mathrm{~ms}$ following TMS onset, after which the signal returned to $\sim 0 \mu \mathrm{V}$. These results are consistent with previous single cell recordings with TMS (Moliadze et al., 2003) that reported reliable action potential detection at approximately $5 \mathrm{~ms}$ following TMS onset.

We also tested whether high frequency TMS induced a modulation in the artifact amplitude or latency. It is possible an increase in amplitude and in duration of the artifact due to the summation of the eddy currents induced by each magnetic pulse in the electrodes, especially when stimuli are delivered at short inter-stimulus intervals. Nevertheless, modulation of the artifact was not
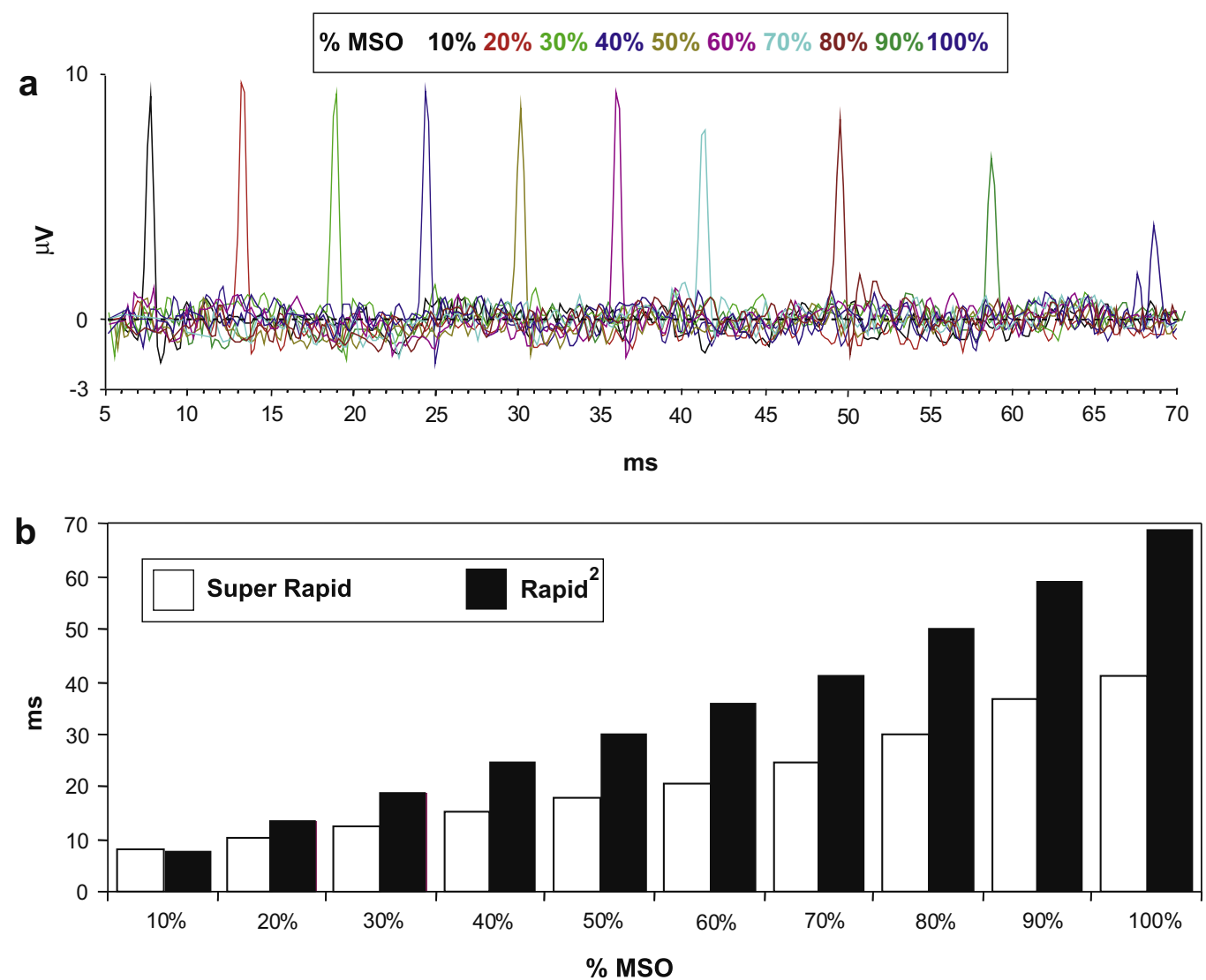

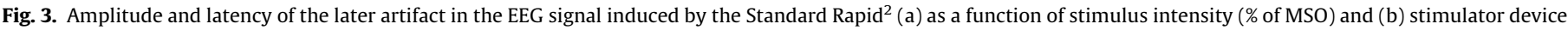
(Magstim super Rapid vs. Rapid ${ }^{2}$ ). 


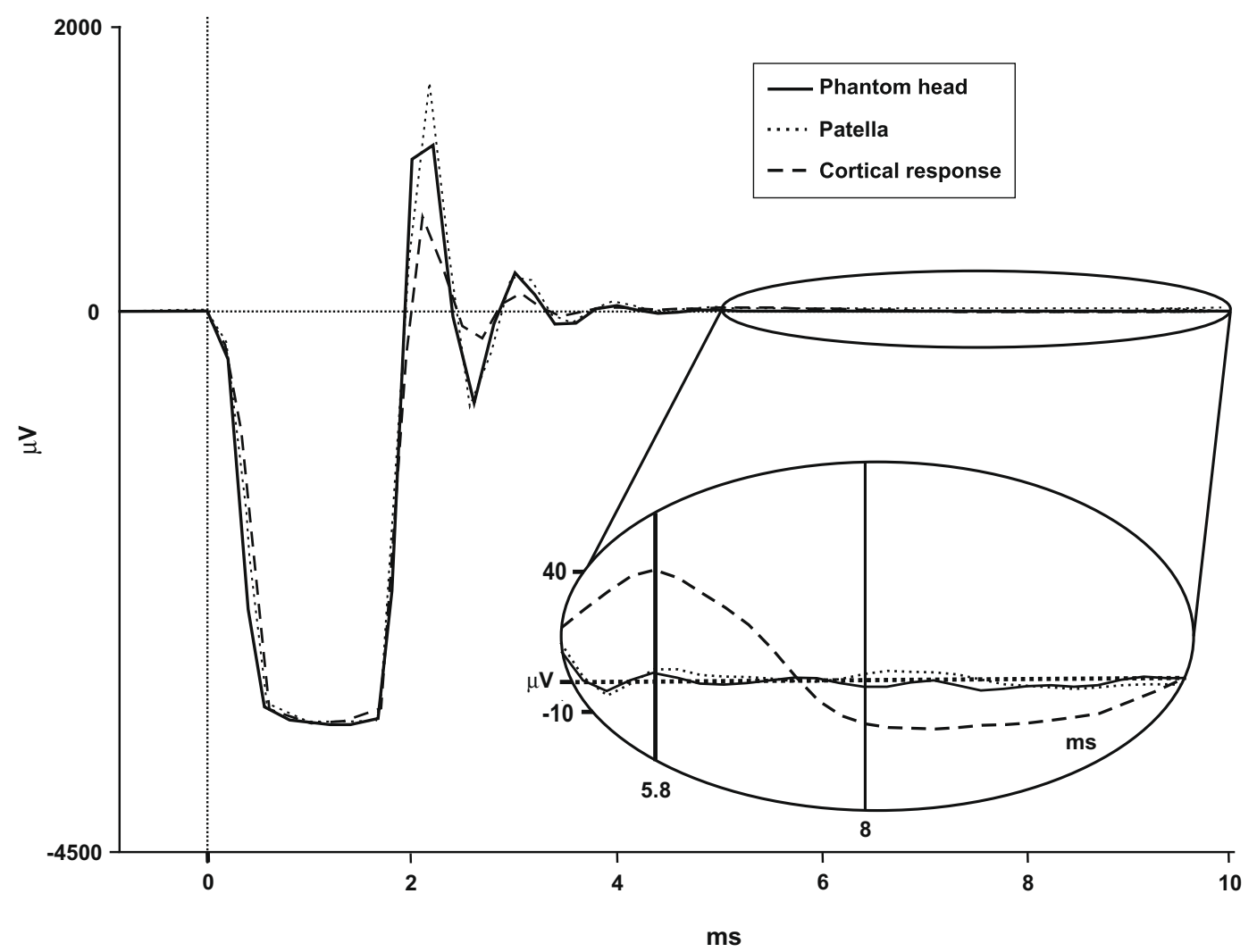

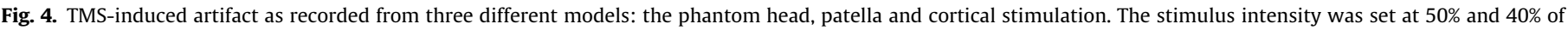

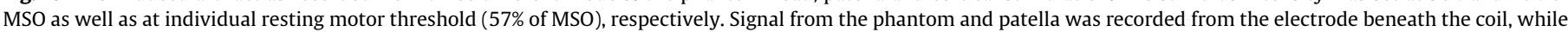
the cortical response was acquired from Cz. The EEG recorded from 5 to $10 \mathrm{~ms}$ is magnified for clear visualization.

induced by this parameter. Intensities or type of coil modulated the artifact amplitude but only with regard to polarity, leaving its length unchanged.

A further consideration is that part of the recorded artifact may also be due to the decay of the stimulus-induced charge differences between the electrode and skin. Such artifacts depend on the capacitive and resistive properties of the skin (see Julkunen et al., 2008). Recording from electrodes over the patella showed that the responses were not different from those recorded in the control condition. Nevertheless, if we consider results of these experiments and we add the common procedures to puncture the skin of the subject in order to reduce impedance, which can reduce the TMS artifact as well (Julkunen et al., 2008), we can conclude that the "electrodeskin" artifact has passed after $5 \mathrm{~ms}$.

It is important to bear in mind that the artifact induced by the TMS pulse does not appear to be the only problem. We showed that the wires should be arranged in an orientation away from the coil or coil cable, regardless of where stimulation takes place on the head. The reorientation of the wires before stimulation can therefore help to record cleaner signals. When recordings are performed with high density EEG, it is recommended that the remaining part of the electrode wires are grouped together toward the amplifier and in opposite direction to the coil cable to avoid additional interference. Moreover, the stimulator recharging can be a further source of artifacts. We found that slowing down the recharging time of the stimulator (i.e., by using only one booster in the case of the Super Rapid device) eliminates this late artifact. Caution should be used when analyzing single subject responses, as this artifact could show the same latency as cortical responses and therefore in some cases this would cause an overlapping of artifactual and real cortical responses. On the other hand, the later artifact is clearly visible as a short transient response, which ap- pears at fixed specific latencies, depending on the stimulation intensity set individually for each subject. To spot this artifact it might be possible to set a phantom head in the laboratory to calibrate its latency for each subject. Nevertheless technical advances can be achieved by inserting a recharging delay circuit in the stimulation machinery.

Another important point to address is the acquisition parameters that should be used. Since the artifact characteristics consist of high frequency variations, the use of a low sampling rate or filters to "clean" the signal will induce a rippling of the signal and therefore a substantial increase in the duration of the artifact. We therefore suggest using a high sampling rate (five times the minimum frequency recorded by the signal that should include the high frequency characteristics of the artifact) and a low pass filter at $1000 \mathrm{~Hz}$. Bonato et al. (2006) showed that by using the same recording system, $1 \mathrm{~Hz}$ rTMS evoked a first cortical response peaking at $8-10 \mathrm{~ms}$ post-stimulus. Small dissimilarities between present results and those reported in the paper by Bonato et al. (2006) could be due to different acquisition parameters (i.e., sampling rate $2.5 \mathrm{kHz}$ and band-pass filter at $0.1-500 \mathrm{~Hz}$ ). As already mentioned, these parameters could produce a decrease in the amplitude of the induced artifact in the first milliseconds after magnetic pulse, which in turn induces a spreading over the EEG signal and thus slowing the signal recovery of some milliseconds. Nevertheless, in the Bonato et al. (2006) paper it is stated that an initial large artifact (4$6 \mathrm{~ms}$ ) was recorded, corresponding to the stimulation site. This is in line with present results since here we demonstrate that the artifact lasts about 5-5.6 ms. Moreover, the cortical response starts to differentiate from patella and phantom head stimulation at $5.8 \mathrm{~ms}$. In a previous paper by Esser et al. (2006), the authors found that stimulation of the motor cortex induced activity at 
the site of stimulation immediately following TMS, with a latency of $5 \mathrm{~ms}$. They analyzed the source localization of this peak and located it to the motor cortex. The differences found in the present experiment after $5.8 \mathrm{~ms}$ resemble the component reported by Esser et al. (2006). Thus, we hypothesize that those responses are not an artifact generated by the TMS system or by the electrode characteristics, even if we cannot exclude other biological artifactual sources.

Care is also needed to avoid additional artifacts during the recording of EEG activity that can be induced by the TMS instrumentation, which may cause misinterpretation of TEP. Further details on combining transcranial stimulation with EEG has been provided previously (for a consensus paper see Siebner et al., 2009).

Determining the duration of a TMS-induced artifact caused by the type of electrode or coil employed is essential for the understanding of the TMS-induced response from different structures of the central nervous system. The possibility to study the early physiological response may have valuable implications for both clinical and experimental purposes. For example, it could provide information regarding the direct cortical response of the stimulated areas and how this correlates with peripheral responses. It has been shown that a good peripheral response (Caramia et al., 1996) correlates with stroke outcome. A better understanding of the early cortical response may therefore have substantial value. Moreover, investigating the cortical response within the first few milliseconds following the delivery of the TMS pulse may allow for a better understanding of TMS-induced effects (i.e., the facilitation and/or inhibition of the cortical activity of a given brain area by TMS).

In conclusion, analyzing the first few milliseconds after TMS onset allowed a characterization of the TMS-induced artifactual response. Thus, the data presented here suggests that it is possible to analyze the cortical evoked response induced by TMS (i.e., TEP) starting at $5 \mathrm{~ms}$ of TMS onset. This enables future research using TMS-EEG co-registration to observe the temporal variation and spatial distribution of the TMS-induced cortical response $5 \mathrm{~ms}$ after the TMS pulse, regardless of the stimulation parameters or electrode characteristics.

\section{Acknowledgements}

We thank Ingmar Gutberlet and Filippo Carducci for useful comments.

\section{References}

Bender S, Basseler $\mathrm{K}$, Sebastian I, Resch $\mathrm{F}$, Kammer $\mathrm{T}$, Oelkers-Ax $\mathrm{R}$, et al. Electroencephalographic response to transcranial magnetic stimulation in children: evidence for giant inhibitory potentials. Ann Neurol 2005;58:58-67.

Bonato C, Miniussi C, Rossini PM. Transcranial magnetic stimulation and cortica evoked potentials: a TMS/EEG co-registration study. Clin Neurophysio 2006;117:1699-707.

Caramia MD, Iani C, Bernardi G. Cerebral plasticity after stroke as revealed by ipsilateral responses to magnetic stimulation. Neuroreport 1996;7:1756-60.

Esser SK, Huber R, Massimini M, Peterson MJ, Ferrarelli F, Tononi G. A direct demonstration of cortical LTP in humans: a combined TMS/EEG study. Brain Res Bull 2006;69:86-94.

Freeston IL, Tozer RC. Impedance imaging using induced currents. Physiol Meas 1995;16(Suppl. 3A):A257-66.

Holder DS, Hanquan Y, Rao A. Some practical biological phantoms for calibrating multifrequency electrical impedance tomography. Physiol Meas 1996;17:A167-77.
Ilmoniemi RJ, Virtanen J, Ruohonen J, Karhu J, Aronen HJ, Naatanen R, et al. Neuronal responses to magnetic stimulation reveal cortical reactivity and connectivity. Neuroreport 1997;8:3537-40.

Iramina K, Maeno M. Measurement of evoked electroencephalography induced by transcranial magnetic stimulation. J Appl Phys 2003:93:6718-20.

Ives JR, Rotenberg A, Poma R, Thut G, Pascual-Leone A. Electroencephalographic recording during transcranial magnetic stimulation in humans and animals. Clin Neurophysiol 2006;117:1870-5.

Julkunen P, Paakkonen A, Hukkanen T, Kononen M, Tiihonen P, Vanhatalo S, et al. Efficient reduction of stimulus artefact in TMS-EEG by epithelial shortcircuiting by mini-punctures. Clin Neurophysiol 2008;119:475-81.

Komssi S, Kahkonen S. The novelty value of the combined use of electroencephalography and transcranial magnetic stimulation for neuroscience research. Brain Res Rev 2006;52:183-92.

Komssi S, Kahkonen S, Ilmoniemi RJ. The effect of stimulus intensity on brain responses evoked by transcranial magnetic stimulation. Hum Brain Mapp 2004;21:154-64.

Litvak V, Komssi S, Scherg M, Hoechstetter K, Classen J, Zaaroor M, et al. Artifact correction and source analysis of early electroencephalographic responses evoked by transcranial magnetic stimulation over primary motor cortex. Neuroimage 2007;37:56-70.

Miniussi C, Thut G. Combining TMS and EEG offers new prospects in cognitive neuroscience. Brain Topogr 2009;2:58-80.

Moliadze V, Zhao Y, Eysel U, Funke K. Effect of transcranial magnetic stimulation on single-unit activity in the cat primary visual cortex. J Physiol 2003;553:665-79.

Morbidi F, Garulli A, Prattichizzo D, Rizzo C, Manganotti P, Rossi S. Off-line removal of TMS-induced artifacts on human electroencephalography by Kalman filter. J Neurosci Methods 2007;162:293-302.

Nelson SO, Trabelsi S, Kays SJ. Dielectric spectroscopy of melons for potential quality sensing. In: Transactions of the ASABE annual international meeting, Rhode Island, June 29-July 2; 2008.

Pascual-Leone A, Dhuna A, Roth BJ, Cohen L, Hallett M. Risk of burns during rapidrate magnetic stimulation in presence of electrodes. Lancet 1990;336:1195-6.

Pascual-Leone A, Houser CM, Reese K, Shotland LI, Grafman J, Sato S, et al. Safety of rapid-rate transcranial magnetic stimulation in normal volunteers. Electroencephalogr Clin Neurophysiol 1993;89:120-30.

Rossi S, Ferro M, Cincotta M, Ulivelli M, Bartalini S, Miniussi C, et al. A real electromagnetic placebo (REMP) device for sham transcranial magnetic stimulation (TMS). Clin Neurophysiol 2007;118:709-16.

Rossini PM, Barker AT, Berardelli A Caramia MD, Caruso G, Cracco RO et al. Noninvasive electrical and magnetic stimulation of the brain, spinal cord and roots: basic principles and procedures for routine clinical application. Report of an IFCN committee. Electroencephalogr Clin Neurophysiol 1994;91:79-92.

Roth BJ, Pascual-Leone A, Cohen LG, Hallett M. The heating of metal electrodes during rapid-rate magnetic stimulation: a possible safety hazard. Electroencephalogr Clin Neurophysiol 1992;85:116-23.

Siebner HR, Bergmann TO, Bestmann S, Massimini M, Johansen-Berg H, Mochizuki $\mathrm{H}$, et al. Consensus paper: combining transcranial stimulation with neuroimaging. Brain Stimul 2009. doi: 10.1016/j.brs.2008.11.002.

Sunaga T, Ikehira H, Furukawa S, Shinkai H, Kobayashi H, Matsumoto Y, et al. Measurement of the electrical properties of human skin and the variation among subjects with certain skin conditions. Phys Med Biol 2002;47:N11-5.

Taylor PC, Nobre AC, Rushworth MF. Subsecond changes in top down control exerted by human medial frontal cortex during conflict and action selection: a combined transcranial magnetic stimulation electroencephalography study. J Neurosci 2007;27:11343-53.

Taylor PC, Walsh V, Eimer M. Combining TMS and EEG to study cognitive function and cortico-cortico interactions. Behav Brain Res 2008;191:141-7.

Thut G, Miniussi C. New insights into rhythmic brain activity from TMS-EEG studies. Trends Cogn Sci 2009;13:182-9.

Thut G, Northoff G, Ives JR, Kamitani Y, Pfennig A, Kampmann F, et al. Effects of single-pulse transcranial magnetic stimulation (TMS) on functional brain activity: a combined event-related TMS and evoked potential study. Clin Neurophysiol 2003;114:2071-80.

Tidswell AT, Bagshaw AP, Holder DS, Yerworth RJ, Eadie L, Murray S, et al. A comparison of headnet electrode arrays for electrical impedance tomography of the human head. Physiol Meas 2003;24:527-44.

Virtanen J, Ruohonen J, Naatanen R, Ilmoniemi RJ. Instrumentation for the measurement of electric brain responses to transcranial magnetic stimulation. Med Biol Eng Comput 1999;37:322-6.

Wassermann EM. Risk and safety of repetitive transcranial magnetic stimulation: report and suggested guidelines from the international workshop on the safety of repetitive transcranial magnetic stimulation, June 5-7, 1996. Electroencephalogr Clin Neurophysiol 1998;108:1-16.

Young IJ, Treen AS, Fixter G, Holden S. Design of solid broadband human tissue stimulant materials. IEE Proc Sci Meas Technol 2002;149:323-8. 